\title{
Railway infrastructure portfolio management in the UK
}

Eliane Marthe Algaard MSc, PhD, CEng, FICE

Director, Route Asset Management, Network Rail, London, UK (corresponding author: eliane.algaard@icloud.com) (Orcid:0000-0002-9756-4288)
Robert David Lines BA, BCom

Managing Director, Project Leaders, London, UK

Six months into its 2014 Railway Upgrade Plan, Anglia Route (UK) was forecasting a shortfall in outputs. A review identified that the delivery plan had not been tested robustly enough for deliverability. The two key root causes were identified as (a) some inertia between the publication of its Strategic Business Plan in January 2013 and the beginning of its Railway Upgrade Plan in April 2014 and (b) the lack of understanding of the key delivery levers and their interdependencies. The solution developed followed two key objectives: (a) a focus on maintaining and renewing the railway to deliver a safe, reliable and sustainable network to the end users (b) without unnecessarily reinventing the wheel. Between 2013/2014 and 2016/2017, Anglia Route increased its capital expenditure delivery from $\mathbf{f 2 5 0}$ million to $\mathbf{f 6 2 4}$ million with no negative impact on train delays. This approach has promoted better communication in the organisation and more control and oversight in delivery.

\section{Introduction}

The railway in the UK is one of the fastest growing in Europe and needs substantial improvements to continue its great success story. Beginning in April 2014 and continuing through March 2019, the Railway Upgrade Plan is Network Rail's response to this tremendous growth and challenge. It is a comprehensive programme of improvements to provide more capacity on the rail network and relieve crowding and congestion.

Anglia Route, one of the eight strategic geographical areas making up the rail network, began in mid-2014 a change programme to improve the timely and cost-effective delivery of its capital infrastructure programme. This paper describes the improvements developed and implemented over the past 3 years.

In the section headed 'Context and adopted strategy', the context and the strategy adopted by Anglia, including the development and implementation of an integrated planning process in partnership with Project Leaders, are described. In the section headed 'Benefits and key successes to date', the benefits of this strategy and key successes to date are reviewed.

\section{Context and adopted strategy}

Anglia Route comprises 1426 track miles $(2295 \mathrm{~km})$ covering the whole of East Anglia and routes into and around London, as described in Figure 1.

Six months into its Railway Upgrade Plan, Anglia Route was forecasting a shortfall in the first year of its 5-year programme. A review identified that the initial delivery plan published in March 2014 had not been tested robustly enough for deliverability and its impact on operational performance in both the short and long term. The disruptive track access required to deliver the published renewal programme had been seriously underestimated, framework contracts were not in place early enough and the works had not been remitted in time to enable delivery to start in April 2014.
The two key root causes were identified as (a) some inertia between the publication of its Strategic Business Plan in January 2013 and the beginning of its Railway Upgrade Plan in April 2014 and $(b)$ the lack of understanding of the key delivery levers and their interdependencies with operational performance. Poor communications between the route asset managers and their infrastructure delivery agent, in part due to a poor understanding of the roles and responsibilities of all the key players, was also acknowledged as a driving factor. These issues were further compounded by the significant growth in passengers and freight traffic over the past 10 years. The increased demand to run more trains and earlier and later trains during weekdays as well as at weekends meant that train and freight operators were more inclined to object to late or significant disruptive access and challenge Network Rail to find creative solutions to deliver infrastructure programmes for the parts of the railway that have difficult access constraints.

These issues are not unique to Anglia Route's Railway Upgrade Plan. In the paper titled 'Operational risk: the focus for major infrastructure', Allport and Ward (2010: p. 123) report a 'dangerous tendency to treat infrastructure development projects whether new build, renewals or upgrades - as if they were activities completely separate from BAU [business-as-usual] operations. Yet such projects deliver beneficial outcomes that justify their investment only if they are subsequently operated efficiently as part of a portfolio of assets'. The key issues identified across sectors by Allport and Ward (2010) are (a) the lack of clear understanding of the interdependencies, $(b)$ poor definitions of the operational objectives and the associated performance criteria, (c) short-termism and a narrow viewpoint, $(d)$ in some cases a dysfunctional project development process and $(e)$ the lack of proactive asset management. The authors conclude that to improve performance, these issues should be taken into consideration when developing an approach to analysing and managing operational risks. In 'Operating risk: the Achilles' heel of major infrastructure projects', Allport (2015) 


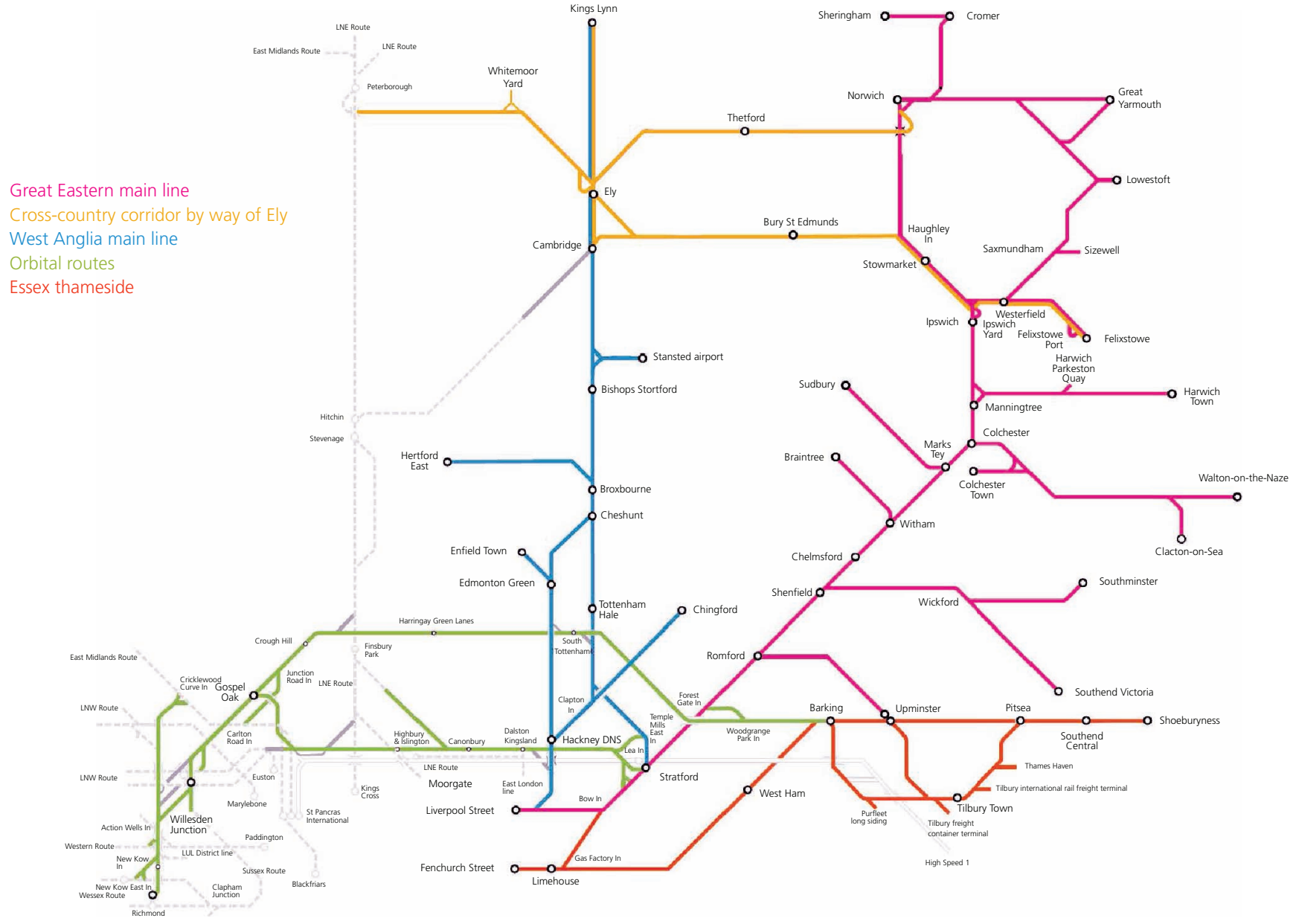

Figure 1. Anglia Route geographical area. LNE, London North Eastern; LNW, London North Western; LUL, London Underground Limited

furthers his case that, in general, transport projects tend to have a poor record in terms of 'achieving operating success' and that a radical change in approach is therefore required to manage operating risks better in line with the encouraging improvements in capital-cost risk management. Allport (2015) identifies that more attention needs to be given to the planning and coordination functions, the project development process and the development of robust business cases based on robust forecasts, as well as the role of the operator.

Gawande (2014) in the BBC Reith lecture The Century of the System eloquently illustrates the way that complex organisations are so interdependent and how extraordinary achievements are possible when meticulous attention is given to every small part. This is also supported by Potts et al. (2016) for the efficient delivery of strategic infrastructure.

Over the past 3 years, Anglia Route has worked towards better management of the operational risks associated with its capital delivery programme through a system approach to problemsolving and better co-operation with its key stakeholders.
The objective was 'to create an organizational context in which cooperation becomes the best choice for each individual' (Morieux and Tollman, 2014: p. 18) in line with the 'six simple rules' promoted by Yves Morieux and to ensure the correct balance between project performance and operational performance are achieved as discussed by Prabhakar (2008).

This improvement programme was founded on the key principles of programme transparency, seamless process flow to fight 'complicatedness' (Morieux and Tollman, 2014), behavioural changes, collaborative working and continuous improvement. The methodology developed was also underpinned by the following two key objectives: $(a)$ a focus on maintaining and renewing the railway to deliver a safe, reliable and sustainable network to the end users $(b)$ without unnecessarily reinventing the wheel. The key changes developed and implemented over the past 3 years were

v the reorganisation of Anglia Route asset-management team and creation of the controlling-mind team from a people's perspective

- the creation of a SharePoint site and a client programme office (CPO) from a governance perspective 
v the development and implementation, in partnership with Project Leaders, of an integrated planning process from a process perspective

n the establishment of an assurance review panel (ARP) from an assurance and stakeholder engagement perspective.

\section{People strategy}

Anglia's route-asset-management team was reorganised in summer 2014 around three core roles: the route asset managers as client, the programme development managers as sponsor and the project managers and planners as the integrated planning team, also known as the controlling mind.

The role of the client, sponsor and client programme office is to ensure effective planning, integration and delivery of its enhancement and renewal projects while operating a railway. They need to balance the portfolio capital work with the route's wider objectives, access and resource constraints, as well as the needs of its key stakeholders. This reorganisation aligns with the clienting guidelines developed by Network Rail and first published in July 2013 and the sponsoring operating model and project life cycle described in the Sponsors' Handbook by Network Rail (2016) published internally in January 2016. The clienting guidelines were also embedded within Anglia Route by way of workshops involving the route asset managers, the sponsors and Anglia delivery partners. The key mindset shift was that by optimising and integrating the plan, key resources were considering the effects on operational performance during and after the planned asset interventions. This mindset drove the geographical packaging of capital works and better consideration of the impact of standalone interventions on other asset disciplines. This led to better operational risk management.

\section{Route client role}

The route client is the directing mind during development and delivery and is accountable for appointing a person to fulfil the sponsor role post transition, integrating, planning and delivering enhancement and renewal projects in conjunction with their operations. This accountability exists throughout the life cycle of the project.

The route client also appoints a representative to discharge the Construction (Design and Management) Regulations 2015 (HMG, 2015) client duties in line with the Network Rail NR/L2/INI/ CP0047 standard, 'Application of the Construction (Design and Management) Regulations to Network Rail construction work' (Network Rail, 2010).

The accountability of the route client was refocused on promoting outcomes that at least protect current performance levels and adhere to long-term asset policy.

\section{Sponsor role}

The sponsor is the single focal point for day-to-day management on behalf of the client. They act on behalf of the clients as the clients' agent, carrying out all the essential management activities, including funding control, management of stakeholder relationships and expectations, gathering of requirements, work acceptance and project close-out. They set the business requirements that the project must deliver using the templates for client and route requirements, which they own, and actively manage delivery of outputs against the business case. This allows the delivery organisation to be accountable to the sponsor for delivery of work to meet the sponsor's stated requirements to agreed scope, cost and schedule within the context of Network Rail's Governance for Railway Investment Projects (Grip) framework.

The restructuring supported the sponsors in encouraging geographically integrated multidiscipline asset interventions that minimise customer disruptions and are in line with good assetmanagement principles.

\section{Integrated planning team}

An integrated planning team was also established to act as a 'controlling mind'. Its role is to take into consideration all the requirements of all the stakeholder groups across the Anglia Route and to maximise the delivery of both renewal volumes and enhancement works, by efficient and coordinated use of the available track access, and to manage the risks associated with the delivery of the planned work within the access time available. The controlling mind had a key objective to translate the strategic plan into an optimal tactical delivery plan (a) safeguarding passenger disruptions to planned minimum and $(b)$ promoting operational performance.

The controlling-mind team is involved in all the stages of the capital delivery process - strategic, tactical, delivery and reporting - and is divided into three subunits: long term, medium term and short term. The framework and processes applied by the controlling mind across these three timelines are further described in the section headed 'Anglia Route integrated planning process'.

\section{Governance strategy}

In addition to the reorganisation described in the section headed 'People strategy', Anglia Route reinforced it governance structure for the delivery of capital works. A SharePoint site and a CPO were set up, and a robust and transparent change-control process was implemented. This change-control process is fully embedded into Network Rail's corporate business planning and investment planning process.

The SharePoint site is used as the core information system for asset management. It contains various lists of data, including the workbank, renewal and enhancement project register, change register, project requirement specifications (PRS) register and possessions plan. It is also used as a repository for assetmanagement documentation.

The workbank forms the live business plan; it shows renewal expenditure by asset type across each financial year. Each 
discipline route asset manager is accountable for specifying the asset interventions defined in the workbank and the ongoing management of that workbank.

The main requirements documents, to be completed by the route asset managers and sponsors in the early of phase of an enhancement or renewal project, are the PRS or the route requirements document as described in Network Rail's PRS process definition document.

Changes affecting the business plan are made using the assetmanagement change-control process. This change process is owned and monitored by the CPO, which is also responsible for the requirement development process and ensures that all parties are fulfilling their commitments. Through this change-control process, project overview is maintained in the project register by the sponsors. Reviews led by the sponsors are held with delivery partners on a periodic basis, and the changes identified are fed into the change register, which reconciles the current plan to the business plan, documenting the reason for the change and type of variance, be that scope or cost-efficiency or another factor. The change register also provides updates for the rolling-forecast process. Changes identified are usually assessed for impact on the Anglia composite reliability index, a regulated output, and within the context of the integrated plan to confirm the availability of resources and access. Figure 2 shows the change-control process implemented.

\section{Anglia Route integrated planning process}

Together with the reorganisation described in the section headed 'People strategy' and the additional governance structure described in the section headed 'Governance strategy', Anglia Route also developed, in partnership with Project Leaders, an integrated planning process facilitated by the Controlling-mind team. The detailed methodology of the controlling mind is shown in Figure 3.

\section{Principles supporting the Anglia Route integrated planning process}

Capital projects in the rail industry are developed and delivered in accordance with the Grip framework, an industry adaptation of the frameworks described in the APM Body of Knowledge (APM, 2012) and Managing Successful Projects with PRINCE2 (Axelos, 2017).

Both these frameworks, however, do not adequately cover the delivery of a complex portfolio of projects in a live and dynamic environment with key interdependencies outside of the project control.

The high-level planning framework developed by Project Leaders and imbedded in the Anglia Route integrated planning process supports the identification at an early stage of all interdependencies, including present and future BAU operational requirements.

This framework is called P4S4P4 - Planning for Safety for Performance - and it ensures that all delivery organisations working on the Anglia Route network have a common level of forward planning ensuring works are integrated and optimised.

The process implemented is complementary to the projectmanagement methodologies described in the APM Body of Knowledge (APM, 2012) and Managing Successful Projects with PRINCE2 (Axelos, 2017), and it closes the gap between integrated and optimised planning and performance outcomes. The key elements of the framework are as follows.

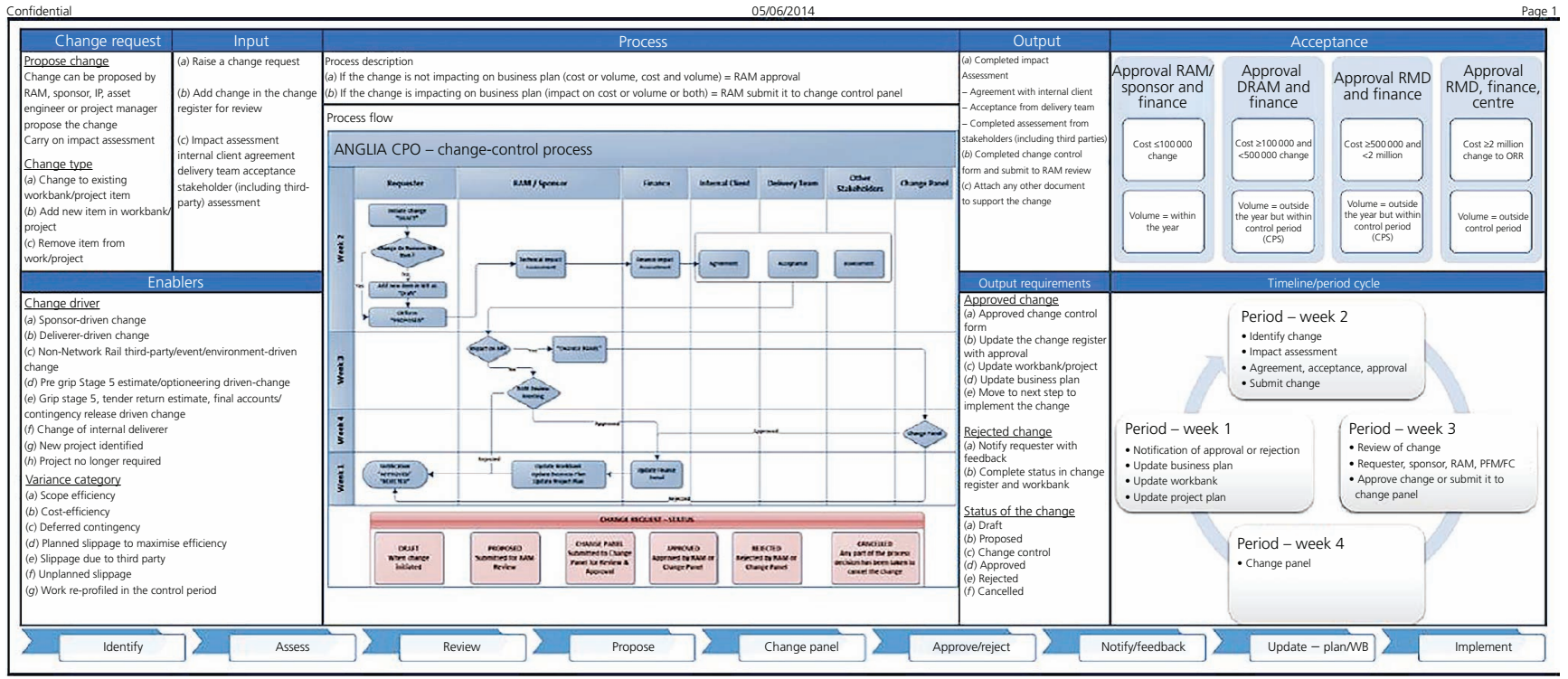

Figure 2. Anglia Route - change-control process. CP5, control period 5; DRAM, director route asset management; FC, financial controller; IP, investment project; ORR, office rail regulation; PFM, performance framework manager; RMD, route managing director 

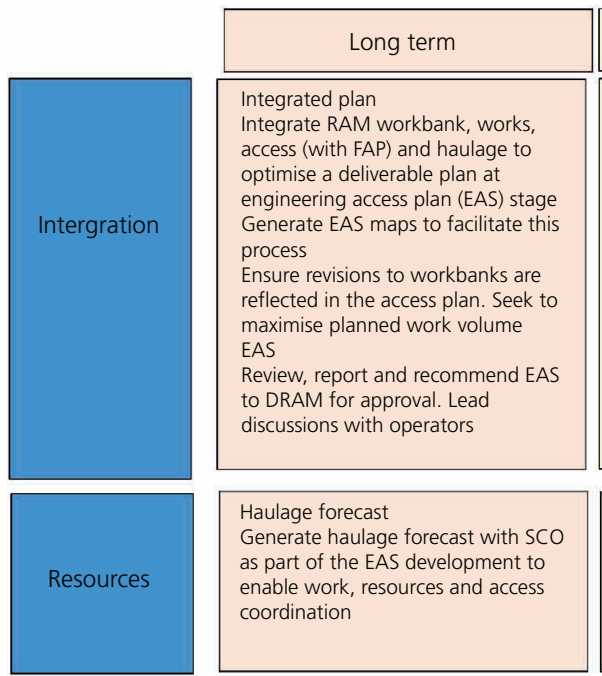

\section{Haulage forecast}

Generate haulage forecast with $\mathrm{SCO}$

as part of the EAS development to

enable work, resources and access

coordination

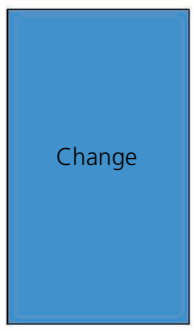

Discruptive access changes submitted after the EAS (but before mediumterm intergration meeting) Organise, chair and record the output for a regular meeting to review, challenge and where appropriate recommend for proposal through planning or reject
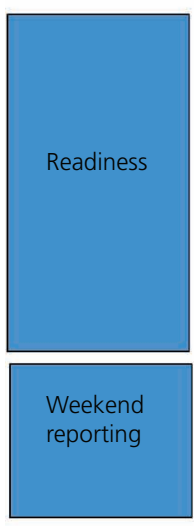

\section{Weekend reporting}

Identify key weekends that warrant

enhanced route reporting,

for example, bank holidays

\section{Request for access to be included} in EAS the EAS based on rests for access in priority maximise volumes delivered

\begin{tabular}{|l|}
\hline \multicolumn{1}{|c|}{ Medium term } \\
\hline Possession and route integration \\
Organise, chair and record the output \\
for an integration meeting to integrate \\
works within pre-identified disruptive \\
possessions and integrate engineering \\
train paths for the whole route \\
before planning and haulage \\
lockdown \\
Assist with efficient allocation of \\
worksite designation at the T-47 \\
integration workshops \\
Organise and chair ad hoc haulage \\
path integration meetings for paths \\
that were unresolved in the T-47 \\
workshops
\end{tabular}
workshops

\section{Haulage}

Obtain confirmation and engineering trains of resource demand and supply at T-47

Resolve clashes or seek executive support for priority decisions

Disruptive access changes submitted after the medium-term integration meeting

Organise, chair and record the output for a regular meeting to review,

challenge and where appropriate

recommend for proposal through

planning or reject. Haulage changes submitted after the medium-term intergration meeting use the medium term follow-up meetings to review engineering train plan changes

Challenge deliverers to

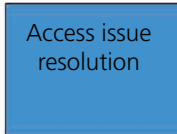

Identification of key route deliverables identity access planned for key route deliverables and agree readiness requirements with delivery team

\section{Weekend reporting}

Identify key weekends that warrant enhanced route reporting, for example, bank holidays

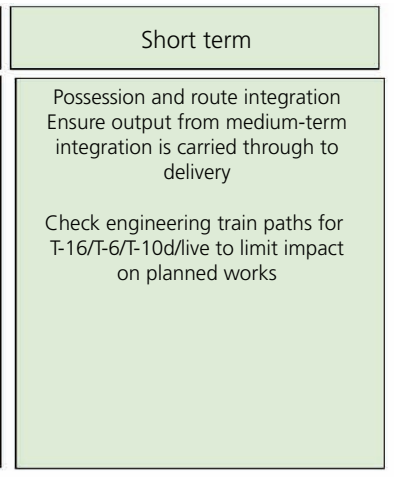

Delivery

For key weekends, obtain evidence from delivery teams that resources are in place

\section{Late changes requiring access change} (past-T-12)

Review and obtain evidence (such as designs, programmes and late scope change) for late change and where appropriate recommend Tor DRAM approval

Key route deliverable readiness organise, chair and record the output from a regular meeting to review and record deliverer readiness for access for key route deliverable (e.g. DWWP red, other intrusive works, key volumes, obtaining evidence of
physical readiness and hazard mitigation) Recommend to executives go/no go from route perspective at T-16/T-6/ T-10d/live

\section{Weekend reporting}

For key weekends that warrant enhanced route reporting, arrange roster and put in place reporting arrangements

Access issue resolution

Access issue resolution planning for specific access issue resolution

Figure 3. Controlling-mind methodology. FAP, freight access planning; SCO, supply chain organisation

(a) Planning Four

(i) logic-linked programme plans are created

(ii) volumes and spend are reflected within the programme

(iii) access and critical resources are reflected within the programme

(iv) integration and multidisciplinary works with others are captured. (b) Safety Four

(i) all readiness reviews (T-) and whiteboards are conducted to an appropriate level

(ii) isolation and access planning is correct and delivered in a timely manner

(iii) incorporating safety by design and build in the programme of works

(iv) safety review of all major works is undertaken. 
(c) Performance Four

(i) keeping to the baseline - slippage is identified early on and mitigated

(ii) keeping to the financial budget

(iii) right-time handback with full scope

(iv) optimal use of the planned access to lessen disruption to the stakeholders.

The role of the integrated planning team is to act as a 'controlling mind' and in effect to implement the P4S4P4 framework described earlier, by following four simple steps: plan, organise, lead and control.

(a) Create the plan in the delivery organisations to a level that is robust and is underpinned by key criteria of resources, access and integration with other delivery groups (long term).

(b) Organise and integrate the various delivery plans by route, asset type and access, to provide the most efficient proposal with the appropriate amount of disruptive access (long term and medium term).

(c) Lead the delivery organisations to satisfy the requirements of running a safe railway and ensuring that maintenance, renewals and enhancements are delivered to meet the key objectives (short term).

(d) Control, monitor and evaluate delivery and be the controlling mind of the integrated plan for the Anglia Route directors (short term).

The preceding methodology allowed for planning at all timescales to be detailed and transparent to all parties and promoted collaboration between the supply chain to deliver collectively. This promotion of an altruistic behaviour between the supply chain demanded compromises against the delivery partner's key objectives for the overall programme and enshrined a system of works approach allowing for each delivery partner to transcend commercial arrangements on the delivery day to ensure overall operational performance and minimum customer disruption.

\section{Long-term controlling mind}

Strategic planning encompasses all activities that result in the successful construction of a timetable of both renewals and enhancements within Anglia Route. To do this, liaison is required with key stakeholders both within Network Rail and externally that is, train- and freight-operating companies and so on. The result of this co-operation is the annual engineering access statement (EAS), which is an industry standard document describing the access required over a specific period.

At this stage, the controlling-mind source access and resource requirements from all deliverers and stakeholders, integrate and optimise through workshops, highlight issues to stakeholders and confirm the prepared plan to all and pull together the formal annual access proposal presented to operators by way of the EAS. This stage saw the beginning of the optimisation of engineering access, and from the outset, a collaborative approach to geographic delivery was achieved.

The controlling-mind team built the long-term plan using a geographically based modelling tool, developed by project leaders. This tool allows categorisation by delivery date, location and deliverer. This then allows the continual cycle of optimisation to occur, which eventually supports an agreed annual access statement and enables a set of weekly access maps to be built.

The final step in the long-term planning phase is ensuring that the use of critical resources is captured. This gives confidence in deliverability and allows deconfliction and balancing of workload, as identified by the critical resource requirements.

Key to the success of the long-term plan is the ability to distil complex information to all the stakeholders in a simple but effective way through the maps previously described.

\section{Medium-term controlling mind}

Following the creation of the EAS, the controlling-mind team coordinates and validates plans from delivery agents to resolve conflicts in access, manpower, haulage and plant requirements. It is at this stage that priorities are applied, which results in some work being advanced, with other lower-priority work being delayed, the objective being a baselined, change-controlled plan against which variations can be tracked and risks documented as explained in the section headed 'Governance strategy'.

The medium-term controlling-mind team leads the 'T-47 weeks' readiness reviews. The purpose of these reviews is to identify potential possession or work site integration issues. Engineering train paths and haulage requirements are also checked during this meeting. Recent changes in asset and system performance are reviewed, and associated additional intervention requirements are assessed to balance project and operational performance.

If conflicts are identified, these are resolved or escalated for prioritisation. This may be due to mutually exclusive deliverability issues or compatibility of works with other routes that border on Anglia. This is particularly relevant when considering the heavy freight traffic on Anglia Route, where running a diversion cross-country for freight may not work if an East or West Coast blockade is happening simultaneously, which does not allow freight to progress beyond the borders of Anglia.

\section{Short-term controlling mind}

The short-term controlling-mind team leads the 'T-12 weeks' readiness reviews. At this stage, the worksites are prioritised based on complexity, route knowledge and the experience of the team. The deliverers are held to account for their proposed work, and assurance deficiencies are highlighted to the Anglia executive team. 
The short-term controlling-mind team also undertakes an integrated safety review from both a logistics and a construction methodology point of view, liaises between all the deliverers for final integration, generates a weekly assurance report with a recommendation on go/ no-go status and also informs decisions on implementation of any contingency plans. The key approach was that there was a single controlling mind who could act as the performance regulator to ensure that no single event would have a detrimental impact to the short-and-long-term objectives, reputationally keeping the infrastructure owner on the right side of regulators and stakeholders. On occasions, the short-term controlling-mind team will attend site to ensure a right-time handback along with a recommendation of which work may need to be cancelled or curtailed to avoid late handback.

\section{Delivery assurance and stakeholder engagement}

To provide an additional level of confidence that capital works activities are delivered within the planned possession times, an ARP was also set up.

The ARP considers work activities that are planned for delivery during specific blockades, bank holiday weekends and the Christmas and New Year holiday period. The ARP is undertaken at defined stages prior to the date of possessions: 12 weeks before (known as T-12), 8 weeks before (T-8), 4 weeks before (T-4) and 2 weeks before (T-2). The ARP consists of representatives from Network Rail, train operators, freight operators, contractors and other delivery organisations and other involved third parties. The ARP is chaired by an independent chairperson.

This process provides the opportunity to demonstrate and test the robustness of the work-planning activities. It also provides key stakeholders the opportunity to gain a level of confidence that there will be a right-time handback at the end of the possession and that there will not be any unplanned disruption as a result of the works.

The ARP identifies issues that need to be resolved before the next stage of the process and provides a quantified level of confidence that the work is being delivered to a robust plan, that all the necessary resources have been identified and are available and that risks that could cause further operational disruption have been identified and are being managed to an acceptable level.

The ARP also ensures that external stakeholders and other relevant third parties have had adequate opportunity to plan for the period of disruption and that they have contingency plans in place as well as clear communication strategies and processes in place to deal with any issues as a result of the disruption.

During these key periods, the controlling-mind team also acts as a dedicated 24/7 progress-reporting team. This enables improved reporting between Anglia Route and its deliverers and therefore more informed management decisions. The dedicated 24/7 progress-reporting team will also provide completion reports detailing the requirements and schedules for any follow-up work or any lessons learned that need to be embedded in future works.

\section{Benefits and key successes to date}

The reorganisation of the route-asset-management team, including the creation of the controlling-mind team, the Anglia asset-management SharePoint site and a CPO, and the development and implementation of a novel integrated planning process as well as a more robust governance structure are driving significant improvements in Anglia Route's capital programme planning and delivery capability in the long, medium and short term.

These changes are also supporting better communication and more collaborative behaviours within Network Rail, its key delivery partners and customers.

\section{Strategic and operational benefits}

The route-asset-management team reorganisation and the creation of the controlling-mind team brought clarity of purpose and clearer focus on Anglia Route's corporate objectives. They enabled a better understanding and ownership of the 5-year programme with the sponsor team now managing with the support of the controlling-mind team the day-to-day renewal programme and therefore allowing the route-asset-management team to focus on the timely delivery of the Control Period 5 (CP5) renewal project remits and the early development of the CP6 renewal workbank. Anglia Route now has a robust 3-year rolling integrated resource-and-access plan and has a better understanding of how key programmes such as Crossrail impact on Anglia objectives and priorities. Between 2013/2014 and 2016/ 2017, Anglia Route increased its capital expenditure delivery from $£ 250$ milliom to $£ 624$ million with no negative impact on train delays. A detailed review by the Department for Transport in 2016 of Anglia's integrated capital programme for 2017-2019 had also identified the programme on a page document illustrating the unprecedented level and complexity of work being undertaken within Anglia Route as 'best in class'. Figure 4 is a snapshot of this programme on a page.

The asset-management SharePoint site together with the CPO has moreover promoted transparency, openness and accountability both within the route-asset-management team and with the delivery partners, and the improved change-control process supported benchmarking of achievements against annual baselines and thus promoted continuous improvement. The short-term delivery reviews have helped promote the development of robust resource-loaded plans, as well as operational contingency plans, and therefore minimise the likelihood and severity of overruns. The number of incidents resulting in a failure of handback on time has decreased from 623 in 2014/2015 to 493 in 2016/2017. This corresponds to a reduction in incidents per million pounds spent from $9 \cdot 4$ to $4 \cdot 5$. This also corresponds to a decrease in the number of delay minutes per million pounds spent from 69 to $33 \mathrm{~min} / £ 1$ million as shown in Figure 5. 


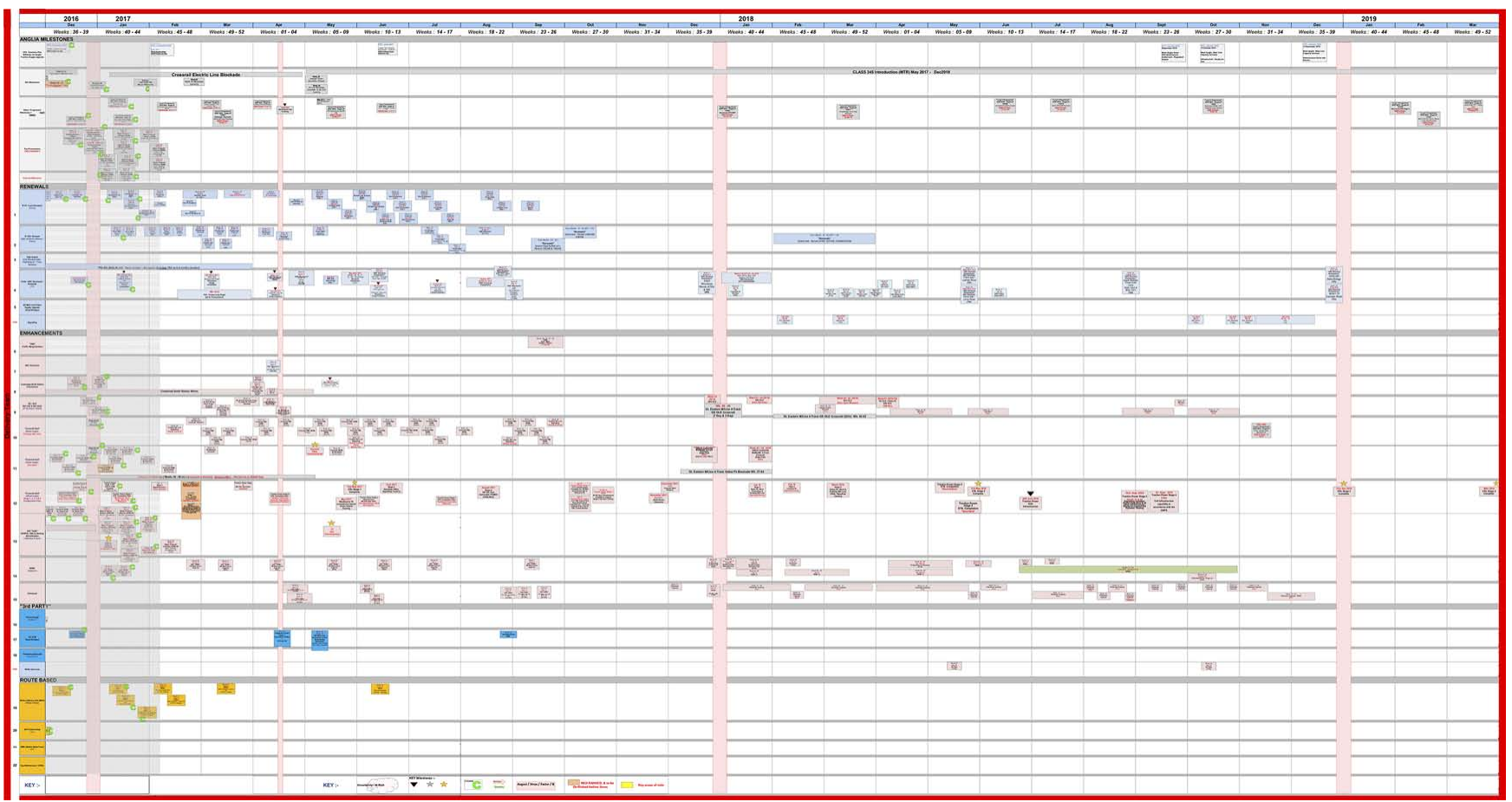

Figure 4. Anglia Route - programme on a page

The long-term controlling-mind process described in the section headed 'Long-term controlling mind' has also led to a better application of the Network Code Decision Criteria and has resulted in a significant reduction in the number of disputes with train and freight operators throughout the iterations of the EAS for 2016/2017, 2017/2018 and 2017/2019.

For example, in August 2016 in a timetable dispute hearing, Anglia Route successfully argued that it had correctly applied the Network Code Decision Criteria to its decision to take the Great
Eastern Main Line Restrictions of Use in weeks 28-30 of 2016/ 2017. This successful dispute hearing was key to the timely completion of Shenfield remodelling and therefore delivery of the Crossrail programme.

Anglia Route is also now able to challenge the validity of access requests within the required timescales to ensure robust timetabling and minimum customer disruption. Anglia Route is able to minimise cancellation costs from contractors by identifying at T-47 incompatibility of works.

Anglia incidents per $\mathrm{f} 1$ million $\quad$ Anglia delay minutes per $\mathrm{f} 1 \mathrm{million}$

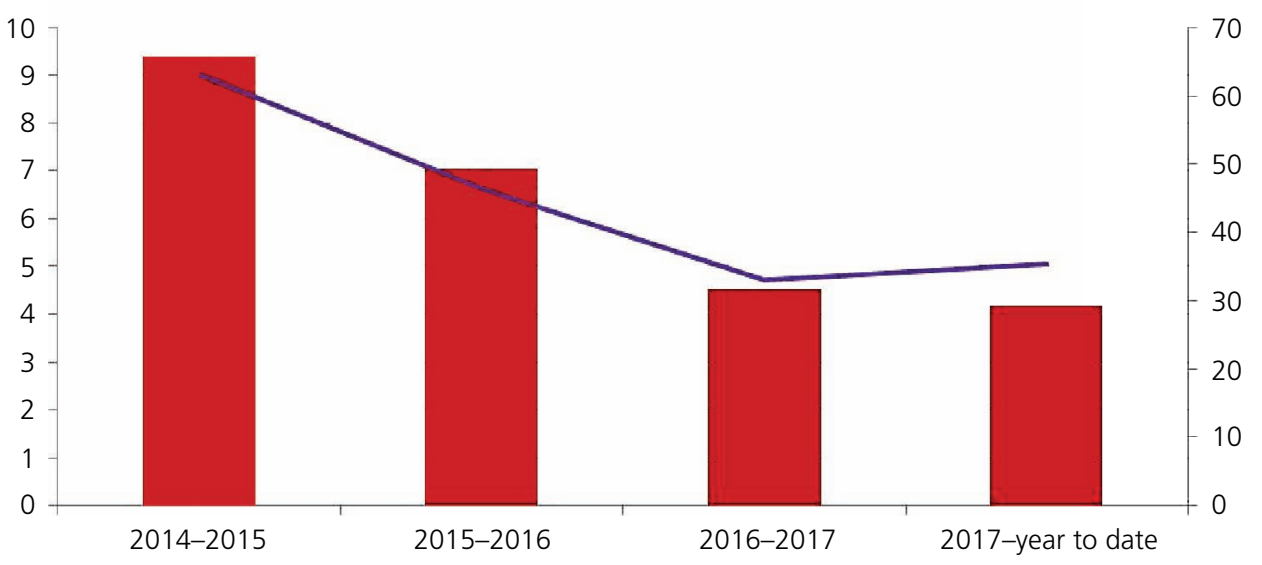

Figure 5. Anglia Route possession-related incidents and delay minutes per $\mathrm{f} 1$ million 
For example, through the T-47 workshop, it was identified that a large number of paths for haulage trains in and out of Whitemoor depot for work throughout Anglia conflicted with possessions on the Ely-March-Peterborough line and an high output ballast cleaning possession at Manningtree between weeks 1 and 24 in 2016. The early identification of these clashes enabled priorities to be agreed with the Track RAM and work to be replanned cost-effectively.

Anglia Route's improved approaches to train movements into and out of possessions not only have identified potential issues in advance but also have contributed to improved understanding by operations and as a result has contributed to an increase in site safety.

\section{Behavioural benefits}

Anglia's integrated master plan approach, enabled by a reorganisation to clarify roles and responsibility, a CPO to promote transparency and the development and implementation of an integrated planning process, is also generating more collaborative working.

A key example of this increase in collaborative working is the Acton Wells Junction Project, completed over Christmas 2015 by the S\&C South Alliance, a Colas-Network Rail partnership. This had started as a relatively simple project: the replacement of 12 items of switches and crossing with a stretch of plain line and a cross-over on a bridge, over the London Underground's central line and another single track. However, it quickly became a complicated project. During the early planning phase, it was identified that the bridge needed substantial work and the Kirov crane required to lift the crossing represented a serious threat to the structural integrity of the bridge. The approach taken combined the bridge repair with the S\&C work, minimising the impact on the availability of the asset for traffic. This junction has strategic importance for freight flow.

The controlling-mind team together with the sponsor actively managed the collaborative effort in planning and delivering the works safely and on time. Engaging all contractors early and creating an integrated plan enabled all issues to be addressed in a collective manner and resolved efficiently. Subsequently, this work was recognised at the 2016 National Rail Awards as a winner for 'team collaboration'. One of the comments from the judges' was as follows: 'most impressive was the motivation of the team and each part of it to compete the work as its main objective, but to deliver the best possible overall industry result'. Figure 6 is a photograph taken during Acton Wells renewals.

The controlling-mind approach and the ARP have promoted better communication between different teams in the organisation and more control and oversight around specific major projects.

They were acknowledged, for example, by Peter Lensink, operations director, Abellio Greater Anglia, for driving 'a

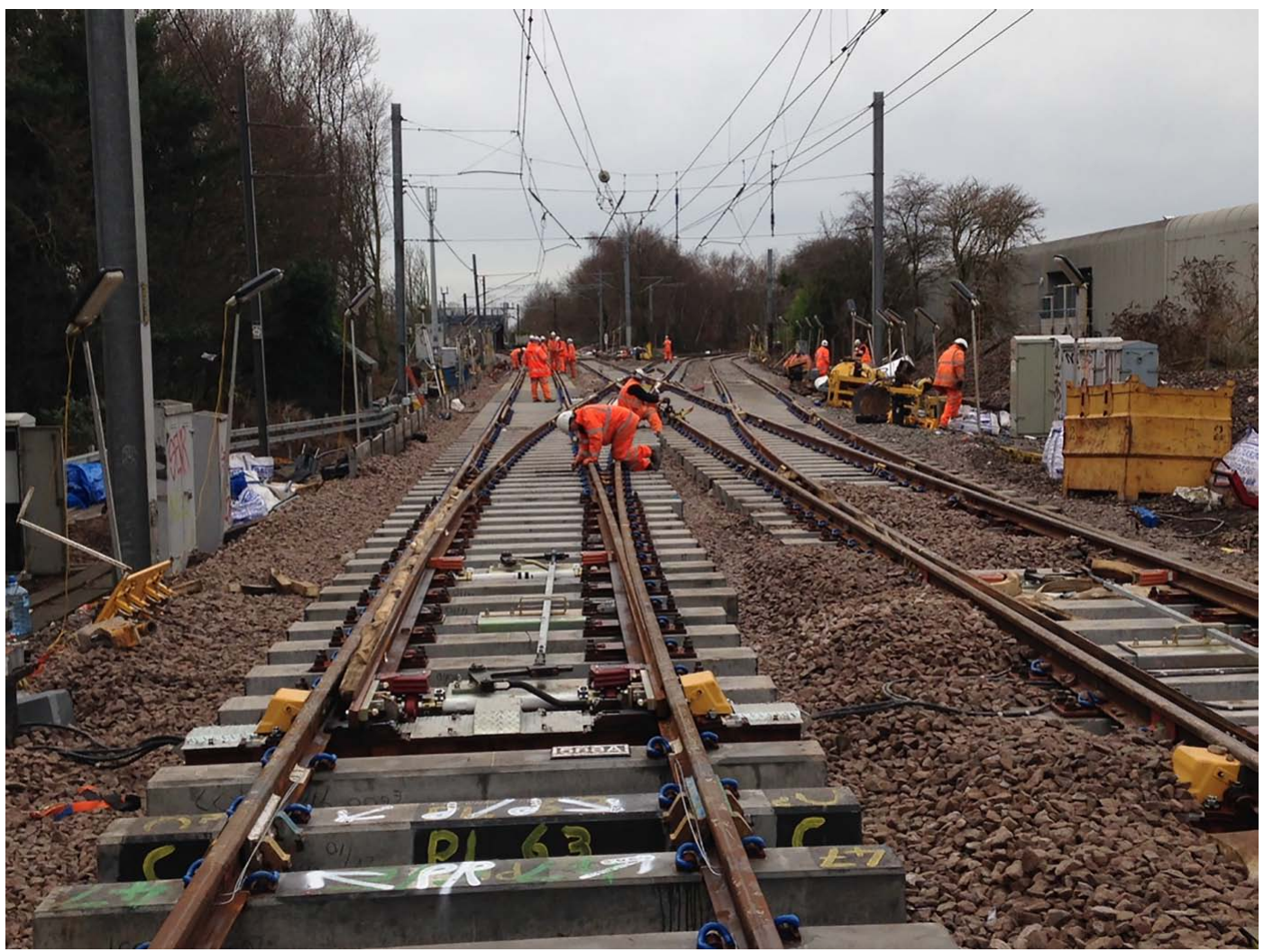

Figure 6. Acton Wells renewals 
reduction in the number of engineering overruns and an improvement in information and communication to us as customer and therewith improved communication with our endusers'. The Anglia Route controlling-mind approach was shortlisted in the 'infrastructure planning' category of the 2017 UK Rail Industry Awards.

\section{Conclusion and next steps}

Through the governance structure, systems and processes being implemented in Anglia, it is progressively becoming a more intelligent client and therefore more confident in the capability of its deliverers. In the past 3 years, over $£ 1429$ million of capital works, both renewals and enhancement, have been delivered successfully.

This approach is followed for the development of the CP6 plans together with an active engagement of the stakeholders both to inform priorities and to increase and sustain transparency around the planning process. This enables Anglia to maintain a delicate balance between stakeholder priorities, funding and engineering access required to deliver the work.

\section{Acknowledgements}

The authors would like to acknowledge all the staff in Anglia Route and the Project Leaders team who contributed to the development and implementation of the approach described in this paper. The authors would like to acknowledge the contribution of Colas Rail and Amco Engineering for the contribution to the success of Acton Wells Bridge \& S\&C Renewal.

\section{REFERENCES}

Allport R (2015) Operating risk: the Achilles' heel of major infrastructure projects. Proceedings of the Institution of Civil Engineers - Civil Engineering 158(3): 130-133, https://doi.org/10.1680/cien.2005.158.3. 130.

Allport RJ and Ward S (2010) Operational risk: the focus for major infrastructure? Proceedings of the Institution of Civil Engineers Management, Procurement and Law 163(3): 121-127, https://doi.org/ 10.1680/mpal.2010.163.3.121.

APM (Association for Project Management) (2012) APM Body of Knowledge, 6th edn. APM Princes Risborough, UK.

Axelos (2017) Managing Successful Projects with PRINCE2®, 6th edn. The Stationery Office London, UK.

Gawande A (2014) The Century of the System: the Reith Lectures 2014: the Future of Medicine, Transcript of Lecture. BBC Radio 4, London, UK.

HMG (Her Majesty's Government) (2015) Construction (Design and Management) Regulations 2015. The Stationery Office, London, UK. Statutory Instrument 2015 No. 51.

Morieux Y and Tollman P (2014) Six Simple Rules: How to Manage Complexity without Getting Complicated. Harvard Business Review Press, Boston, MA, USA.

Network Rail (2010) NR/L2/INI/CP0047 Issue 4: Application of the Construction (Design and Management) Regulations to Network Rail construction work. Network Rail, London, UK

Network Rail (2016) Sponsors' Handbook. Network Rail, London, UK. Potts M, McDermott P and Stephenson A (2016) A systems approach to strategic infrastructure delivery. Proceedings of the Institution of Civil Engineers - Management, Procurement and Law 169(3): 95-105, https://doi.org/10.1680/jmapl.15.00026.

Prabhakar GP (2008) Projects and their management: a literature review. International Journal of Business and Management 3(8): 3-9, https:// doi.org/10.5539/ijbm.v3n8p3.

\section{How can you contribute?}

To discuss this paper, please submit up to 500 words to the editor at journals@ice.org.uk. Your contribution will be forwarded to the author(s) for a reply and, if considered appropriate by the editorial board, it will be published as a discussion in a future issue of the journal. 